\title{
Identificación del quehacer en la gestión organizacional a la luz de la responsabilidad social universitaria
}

\section{Identification of organizational management tasks in the light of the university social responsibility}

\author{
Sara C. Velázquez-Velázquez ${ }^{a}$, Clara Carlson-Morales ${ }^{b}$
}

\begin{abstract}
:
The objective was to identify the elements of the work of an educational institution in the area of organizational management impact, in order to fulfill the purpose in the context of University Social Responsibility, the method was that of documentary review of descriptive and exploratory type, for this we proceeded to consult and analyze the self-diagnosis Matrix URSULA (Latin American University Social Responsibility Unit), PRME (Principles for Responsible Education in Management and the Rubric of the distinctive of University Social Responsibility by ANFECA, as well as ISO 21001: 2018 Management Systems for Educational Organizations. Among the main results we found the acknowledgement that there are several tools for self-diagnosis of RSU and that their application is feasible, so we conclude on the importance of working through indicators that allow continuous improvement and generate positive impacts on the management of the institution.
\end{abstract}

Keywords:

University Social Responsibility, Self-diagnosis, Organizational Management

\section{Resumen:}

El objetivo fue identificar los elementos del quehacer de una institución educativa en el área de impacto de gestión organizacional, con el fin de cumplir con el propósito en el contexto de la Responsabilidad Social Universitaria, el método fue el de revisión documental de tipo descriptivo exploratorio, para ello se procedió a consultar y analizar la Matriz de autodiagnóstico URSULA (Unidad de Responsabilidad Social Universitaria Latinoamericana), PRME (Principios para la Educación Responsable en Gestión y la Rúbrica del distintivo de Responsabilidad Social Universitaria por parte de ANFECA, así como la ISO 21001:2018 Sistemas de Gestión para Organizaciones Educativas. Entre los principales resultados encontramos el reconocer que existen diversas herramientas de autodiagnóstico de RSU y que son viable su aplicación, por lo que concluimos en la importancia de trabajar por medio de indicadores que permitan la mejora continua y generar impactos positivos en la gestión de la institución.

\section{Palabras Clave:}

Responsabilidad Social Universitaria, Autodiagnóstico, Gestión Organizacional

\section{Introducción}

Las instituciones educativas forman parte fundamental en el desarrollo de la sociedad, puesto que contribuyen en la educación y formación integral de sus educandos, para ello debe dar ejemplo en la generación de impactos positivos para mejorar la sociedad.

\section{Desarrollo}

Recobra importancia la gestión organizacional a luz de la Responsabilidad Social Universitaria, como política de calidad del desempeño de todas las áreas que conforman la comunidad universitaria. Por lo que se procede a realizar un diagrama del conocimiento y posteriormente el diseño de un autodiagnóstico que considera el ámbito de Gestión de la Organización, por medio de un análisis, recopilación y reestructuración de los diversos indicadores recopilados en los siguientes documentos:

\footnotetext{
a Autor de Correspondencia, Universidad Linda Vista, https://orcid.org/0000-0001-9030-8938, Email: sara.velazquez@ulv.edu.mx

b Universidad Linda Vista, https://orcid.org/0000-0002-9752-8033, Email: clarita.carlson@ulv.edu.mx
} 
Matriz URSULA (2019): La Unidad de Responsabilidad Social Universitaria Latinoamericana en el documento MLRSU-004-A1, revisión 0.1, ámbito de acción 1/Metas e indicadores de RSU en el área de impacto de gestión organizacional. La matriz está formada por tres metas: 1. Buen clima laboral, con siete indicadores. 2. Campus Sostenible con cinco indicadores, 3. Ética, transparencia e Inclusión con seis indicadores. Se tomaron todos los indicadores a considerar en el diseño del autodiagnóstico [1].

Rúbrica ANFECA (2018): Considerando lo que mide la Asociación Nacional de Facultades y Escuelas de Contaduría y Administración en el eje de impacto organizacional y ambiental en los seis principios: 1. Compromiso ético con tres indicadores, 2. Transparencia, rendición de cuentas y no corrupción con tres indicadores, 3. Respeto a los derechos humanos, 4. Cultura de la legalidad y respeto a las normas con siete indicadores, 5 . Participación ciudadana, 6. Sustentabilidad, los últimos cuatro principios con un indicador cada uno [2].

Gestión (PRME): Organización que promueve la sostenibilidad, la incorporación de los valores universales en los programas y la investigación y principios para la educación responsable se analizó el área Liderazgo y Estrategia Institucional que se conforma de los siguientes aspectos generales: 1. Gobierno institucional con tres sub aspectos: marco general, conflicto de interés y Equidad de género, 2. Estrategia institucional con tres sub aspectos: Integración con la Sostenibilidad, Grupos de interés y propósito, 3. Control de la Operación con dos sub aspectos: Equipo y seguimiento, 4. Reporte y rendición de cuentas con dos sub aspectos: Reporte, verificación y comunicación, 5. Mejora e Innovación con dos sub aspectos: Acciones y desarrollo, 6. Impacto: Docencia y extensión [3].

ISO 21001-2018 Sistema de Gestión para las Organizaciones Educativas 7. Este sistema está basado en los siguientes enfoques: al cliente, liderazgo, compromiso de las personas, enfoque a procesos, mejora, toma de decisiones basada en la evidencia y gestión de las relaciones, responsabilidad social corporativa, accesibilidad y equidad, conducta ética, y seguridad y protección de datos [4].
Como resultado de este análisis y recopilación de indicadores, se formó un solo documento de Autodiagnóstico de la Gestión Organizacional conformado por ocho categorías: Buen clima laboral, Compromiso ético, Cultura de la legalidad y respeto a las normas, Respeto a los derechos humanos, Transparencia, Rendición de cuenta y no corrupción, Sustentabilidad, Mejora e Innovación e Impacto. Cada una con sus respectivas metas e indicadores de logro.

\section{Conclusión}

Se logró identificar por medio de la consulta de los diversos materiales, áreas de oportunidad que faciliten el cumplimiento del propósito de la Responsabilidad Social Universitaria desde la gestión organizacional

\section{Referencias}

[1] Unión de Responsabilidad Social Universitaria Latinoamericana. (2016). Matriz de autodiagnóstico RSU - URSULA 2021. Español. Recuperado de: https://unionursula.org/herramientas-rsu/

[2] Asociación Nacional de Facultades y Escuelas de Contaduría y Administración (ANFECA), (2018). Formato para el llenado del autodiagnóstico y plan de mejora.xlsx, para la obtención del distintivo de responsabilidad social universitaria.

[3] Gómez Pugarín, D., Guevara, G., Yepes López, G., Martí Noguera, J. y Díaz Cáceres, N. (2018). Sistema de Indicadores de Responsabilidad Social Universitaria para el Informe de Progreso PRME. Colombia: Corporación Red Local del pacto Global en Colombia

[4] Organización Internacional de Normalización ISO. ISO 21001:2018. Organizaciones educativas. Sistemas de gestión para las organizaciones educativas. Requisitos con orientación para su uso. Ginebra, Suiza: Organización Internacional de Normalización (ISO); 2018. 


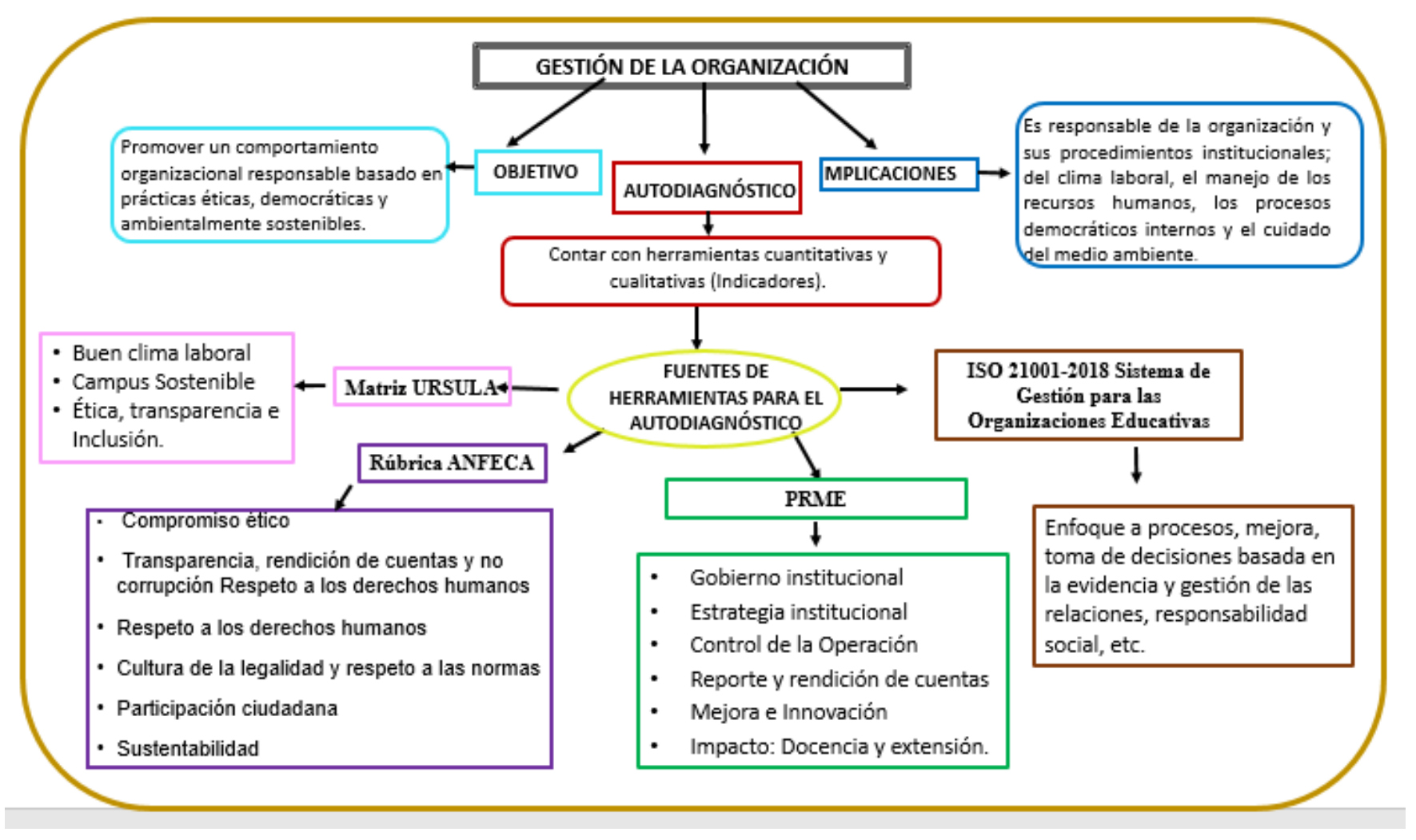

Figura 1. Gestión Organizacional

Nota: Elaboración propia, en base a URSULA (2021), ANFECA (2018), PRIME (2018), ISO 21001:2018. Organizaciones Educativas 